\title{
Planetary Rovers' Wheel Sinkage Detection Based on Wheel-Soil Boundary
}

\author{
Lv Fengtian ${ }^{1, a}$, Li Nan ${ }^{1, b, *}$, Gao Haibo, ${ }^{1, \mathrm{c}}$, Ding Liang ${ }^{1, \mathrm{~d}}$, Liu Zhen ${ }^{1, \mathrm{e}}$, \\ Deng Zongquan ${ }^{1, \mathrm{f}}$, Song Xingguo ${ }^{1, \mathrm{~g}}$ Yan Bing $^{2, \mathrm{~h}}$ \\ ${ }^{1}$ State Key Laboratory of Robotics and System of HIT, China \\ ${ }^{2}$ Beijing Institute of Astronautical Systems Engineering, China \\ ahitlft@163.com, ${ }^{b}$ Inlinanln@126.com (corresponding author's email), ${ }^{c}$ \\ gaohaibo@hit.edu.cn, ${ }^{\mathrm{d}}$ liangding@hit.edu.cn, ${ }^{\mathrm{e}}$ liuzhen_hit@163.com, ${ }^{\mathrm{f}}$ dengzq@ @hit.edu.cn, ${ }^{9} \mathrm{xgsong}$
} @hotmail.com, hyanice@163.com

Keywords: planetary rovers; wheel-soil interaction; wheel-soil boundary; sinkage; feature extraction

\begin{abstract}
The detection of wheel sinkage has great significance for rover mobility optimization control and prevention of wheel sinking. A new wheel sinkage detection method is proposed based on planetary rovers' wheel-soil boundary. The model of wheel sinkage calculation is built. The machine vision method is proposed to extract wheel-soil boundary. Wheel-soil interaction image is processed into binary image, and the wheel-soil boundary is extracted according to its morphological features. The wheel sinkage depth, entrance angle and departure angle are calculated through the model of wheel sinkage calculation. The method's applicability has been validated by experiments under various terrain conditions, which are flat terrain, raised terrain, hollow terrain, and rough terrain. Accuracy tests are done with the flat terrain, the results of the experiments indicate that the relative errors of the sinkage depth are around $10 \%$ and the relative errors of terrain interface angles are around $5 \%$, when the actual sinkage depth is above $5 \mathrm{~mm}$.
\end{abstract}

\section{Introduction}

Rovers need to perform challenging mobility in rough terrain for the planetary exploration missions. Wheel-terrain plays an important role in rovers' mobility ${ }^{[1]}$. In general, comparing with hard terrain, the soft terrain produces worse traction and thus less effective mobility.

Wheel sinkage is a key variable in estimating and predicting wheel-terrain interaction ${ }^{[2]}$. The large wheel sinkage may cause traction loss, and the rovers move difficultly. For example, the sprint and opportunity rovers had sunk into soil many times. With detecting to know wheel sinkage, the wheel torque of rovers could be modulated to improve traction, or its motion plan is revised to avoid potentially hazardous highly-deformable terrain. Wheel sinkage is also an important parameter for terrain identification and classification ${ }^{[3]}$.

Previous research measures sinkage of the wheel relative to another wheel. However, it can not know the absolute sinkage relative to terrain. Tradition methods apply wheel-soil interaction model to estimate wheel sinkage. The wheel sinkage detection through machine vision method is a non-contact measurement manner, it can directly detect the wheel sinkage. There are some methods based on gray image in paper [4-9], the wheel-soil interaction points which could be used to calculate entrance angle and departure angle are extracted according to that the wheel and the terrain are in different gray level. Those methods can detect wheel sinkage efficiently, but they are sensitive to illumination intensity and shadow in the image. Hegde proposed a wheel sinkage detection method based on color image processing ${ }^{[10]}$. Some areas which are irrelevant to sinkage are removed, the image is cut into several images through normalization image segmentation method. The terrain region is extracted and the wheel-soil interaction boundary and points are all acquired to calculate the terrain interaction angles and the sinkage depth.

Most of their sinkage detection methods used the entrance or departure angle to calculate sinkage with wheel radius. It has not considered the variation of terrain which is considered in this paper. The 
position of camera relative to the wheel is fixed in our study. Wheel sinkage detection will be studied in the future when the position is changing.

\section{Wheel-soil boundary Extraction and Sinkage Calculation}

Wheel Sinkage Calculation Model. On flat terrain, the sinkage depth is the distance from the lowest point of the wheel to the terrain horizontal. For rough terrain, we build the wheel sinkage calculation model as shown in Fig. $1 . O$ is the wheel's center, $r$ is the wheel's radius, $\theta_{1}$ is the angle between $O A$ and the vertical direction and $\theta_{2}$ is the angle between $O B$ and the vertical direction. The equivalent line is constructed through using the least squares fitting. The origin of coordinates $\Sigma O-x y$ locates in the center of the wheel, with $x$ axis directing to the horizontal direction and $y$ axis directing to the minus vertical direction. The wheel sinkage depth is $z_{0}=r$-OC. The entrance angle and the departure angle are $\theta_{1}$ and $\theta_{2}$.

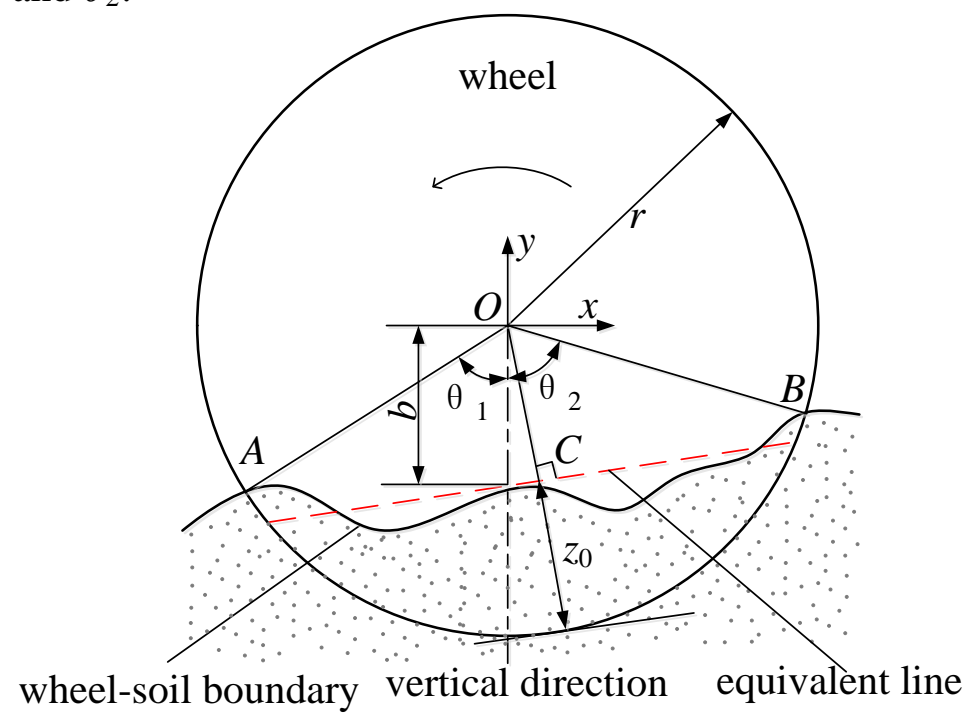

Fig. 1. The sinkage calculation model

The equivalent line is $y=k x+b$, the wheel-soil boundary line's points are $y_{i}=f\left(x_{i}\right)(i=1 \ldots n$.). the sinkage depth is

$$
z_{0}=r-O C=r-\frac{|b|}{\sqrt{k^{2}+1}} .
$$

The equation's parameters $k$ and $b$ are respectively described as $k=\left[n \Sigma y_{i} x_{i}-\Sigma y_{i} \Sigma x_{i}\right] /\left[n \Sigma x_{i}^{2}-\left(\Sigma x_{i}\right)^{2}\right]$ and $b=\left[\Sigma y_{i} \Sigma x_{i}^{2}-\Sigma x_{i} \Sigma y_{i} x_{i}\right] /\left[n \Sigma x_{i}^{2}-\left(\Sigma x_{i}\right)^{2}\right]$.

The coordinates of $A$ and $B$ are expressed as $\left(x_{\mathrm{A}}, y_{\mathrm{A}}\right),\left(x_{\mathrm{B}}, y_{\mathrm{B}}\right)$ in Fig. 1 . The terrain interaction angles (TIAs) are the entrance angle and departure angle, they can be described as

$$
\theta_{1}=\left\{\begin{array}{cc}
\pi / 2-\arctan \left(y_{\mathrm{A}} / x_{\mathrm{A}}\right) & x_{\mathrm{A}} \neq 0 \\
0 & x_{\mathrm{A}}=0
\end{array},\right.
$$

and

$$
\theta_{2}=\left\{\begin{array}{cc}
\pi / 2+\arctan \left(y_{\mathrm{B}} / x_{\mathrm{B}}\right) & x_{\mathrm{B}} \neq 0 \\
0 & x_{\mathrm{B}}=0
\end{array} .\right.
$$

Wheel-soil boundary Extraction Method. The wheel-soil interaction area image is a color image. This paper does the image segmentation based on color features. Transform the image color 
space form RGB to HSI, I component is not relative to $\mathrm{H}$ and $\mathrm{S}$. The camera's position relative to the wheel is fixed, so the wheel confidence area shown in Fig. 2 can be divided uniformly, the wheel confidence area rectangle size is $a_{1} \times b_{1}$ and its upper left point's pixel coordinate is $\left(m_{1}, n_{1}\right)$. Calculate the average value $H_{\mathrm{w}}$ and $S_{\mathrm{w}}$ of $\mathrm{H}$ and $\mathrm{S}$ components in the rectangle area. $H_{\mathrm{w}}$ and $S_{\mathrm{w}}$ are

$$
H_{\mathrm{w}}=\left(\sum_{i=m_{1}}^{i=m_{1}+a_{1}-1} \sum_{j=n_{1}}^{j=n_{1}+b_{1}-1} H(i, j)\right) /\left(a_{1} \times b_{1}\right)
$$

and

$$
S_{\mathrm{w}}=\left(\sum_{i=m_{1}}^{i=m_{1}+a_{1}-1} \sum_{j=n_{1}}^{j=n_{1}+b_{1}-1} S(i, j)\right) /\left(a_{1} \times b_{1}\right) .
$$

The value scope of $\mathrm{H}$ component $H_{\mathrm{a}}$ and $H_{\mathrm{d}}$ for image segmentation can be described as $H_{\mathrm{a}}=$ $P_{\mathrm{a}} H_{\mathrm{w}}$, and $H_{\mathrm{a}}=P_{\mathrm{a}} H_{\mathrm{w}} . P_{\mathrm{a}}$ and $P_{\mathrm{d}}$ are respectively the upper limit and lower limit percentage. The value scope of $S$ component $S_{\mathrm{a}}$ and $S_{\mathrm{d}}$ can be calculated through the same method. Process the image to binary image according to the value scope of $\mathrm{H}$ and $\mathrm{S}$. The further processing for binary image includes dilation, erosion, area filtering and so on. The binary image is shown in Fig. 3.

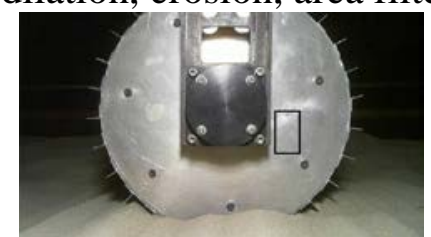

Fig. 2. The wheel confidence area.

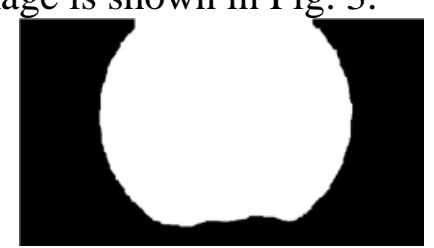

Fig. 3. The binary image.

The outline of wheel area shown in Fig. 4 can be extracted from the binary image. Transform the outline into three-dimensional Hough space to extract the wheel rim line and get the wheel's center and radius. If the distance between points of the outline with the point $O$ is less $r$, the points are in the wheel-soil boundary. According to this feature, the wheel-soil boundary as shown in Fig. 5 is extracted, so we can know the coordinates of its points.

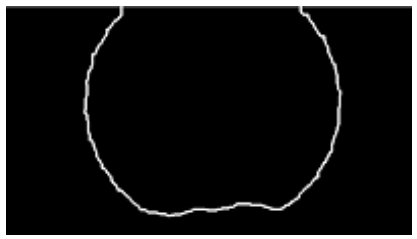

Fig. 4. The wheel's outline.

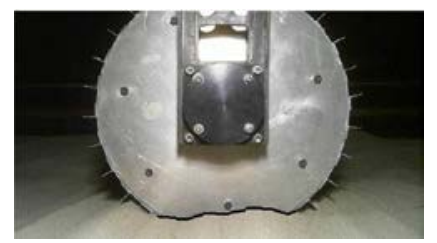

Fig. 5. The results of extraction.

The wheel-soil boundary line points' coordinates are the inputs of the parameters $k$ and $b$ in Eq. 1, then the sinkage $z_{0}$ can be calculated through Eq. 1. TIAs are calculated through Eq. 2 and Eq. 3 with the coordinates of $A$ and $B$.

The Processes of Sinkage Detection. The processes of sinkage detection are as follows:

(1) Acquire a wheel-soil interaction area image from the camera and divide the wheel confidence area in the image.

(2) Transform the image color space from RGB to HSI.

(3) Calculate the value of $\mathrm{H}$ and $\mathrm{S}$ components of wheel confidence area. Acquire the respective value range of $\mathrm{H}$ and $\mathrm{S}$ for image segmentation, and then execute the image binarization processing.

(4) Extract the outline of the wheel area. Extract the center $O$ and radius $r$ of the wheel rim based on the outline of the wheel area. Extract the wheel-soil boundary based on its features.

(5) Use the Equation Eq. 1, Eq. 2 and Eq. 3 to calculate the sinkage depth and TIAs. 


\section{Experiments Validation}

Experiment Equipment. The wheel-soil interaction test platform with vision collection system is shown in Fig. 6. The three motors on the platform are drive motor, turning motor, dragging motor, the sensors on the platform are torque sensor, displacement sensor, six-axis force / torque sensor, and so on. The drive motor drives the wheel forward, the dragging motor is used to simulate the rover body's movement. It can be controlled that the wheel moves with different slip ratios through controlling the two motors in different rotate speed. The displacement sensor is used to measure the sinkage depth, its base level is the flat terrain's horizontal. The counter weight can be used to adjust the wheel's vertical loads, the actual loads are read from six-axis force / torque sensor. The vision collection system is used to gain the wheel-soil interaction area image. The wheel's radius is $140 \mathrm{~mm}$ and width is $150 \mathrm{~mm}$. The wheel with 28 lugs that are $15 \mathrm{~mm}$ high is shown in Fig. 7.

Table 1 is the parameters of the soil provided by Shanghai Academy of Spaceflight Technology. The $k_{\mathrm{c}}\left(\mathrm{kpa} / \mathrm{m}^{\mathrm{n}+1}\right)$ is the cohesive modulus of soil, $k_{\varphi}\left(\mathrm{kpa} / \mathrm{m}^{\mathrm{n}}\right)$ is the frictional modulus of soil, $n$ is the exponent of the sinkage, $c(\mathrm{kpa})$ is the cohesion of soil, $\varphi\left({ }^{\circ}\right)$ is the internal friction angle of soil.

Table 1. The parameters of the soil

\begin{tabular}{cccccc}
\hline$\rho[\mathrm{kg} / \mathrm{m} 3]$ & $k_{\mathrm{c}}\left[\mathrm{kpa} / \mathrm{m}^{\mathrm{n}-1}\right]$ & $k_{\varphi}\left[\mathrm{kpa} / \mathrm{m}^{\mathrm{n}}\right]$ & $n$ & $c[\mathrm{kpa}]$ & $\varphi\left[^{\circ}\right]$ \\
\hline 1.60 & -20.7 & 1594.8 & 0.79 & 0.46 & 38.1 \\
\hline
\end{tabular}

Rovers' wheel vertical load is different when the rover moves on different planet. The $120 \mathrm{~kg}$ lunar rover wheel average vertical load is $33 \mathrm{~N}$ and the $200 \mathrm{~kg}$ mars rover wheel average vertical load is $131 \mathrm{~N}$. The vertical loads can be set as $25-150 \mathrm{~N}$ in the experiments, the representative vertical load is $90 \mathrm{~N}$.

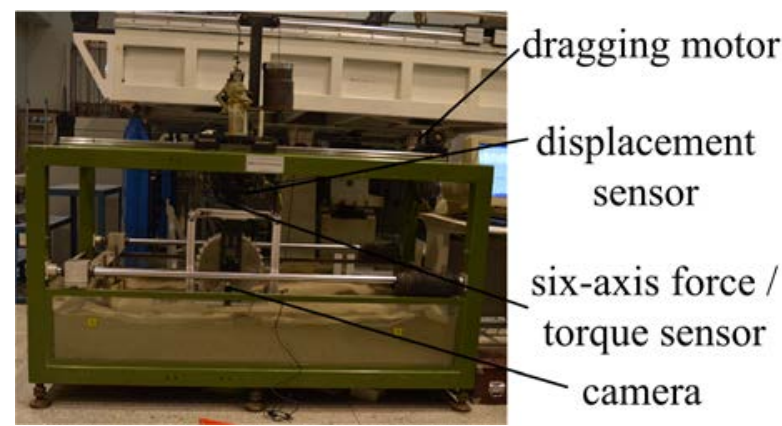

Fig. 6. Wheels - soil interaction test platform.

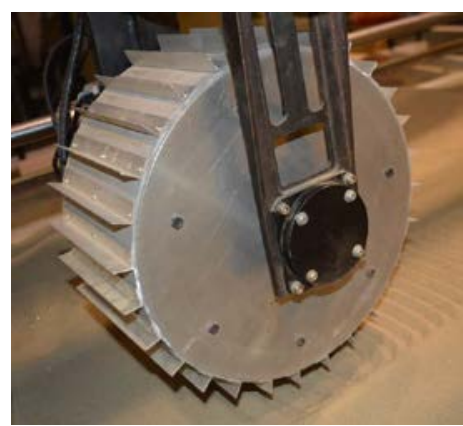

Fig. 7. The wheel.

Terrain adaptability testing experiments. The terrain can be classified to four conditions: flat terrain (FT), raised terrain (RT), hollow terrain (HT), and rough terrain (RT). In the experiments, Wheel speed is $10 \mathrm{~mm} / \mathrm{s}$, the vertical load is $90 \mathrm{~N}$, and the slip ratios are $0.2,0.4$, and 0.6 in each terrain condition. There are 12 experiments.

In the experiments, the displacement sensor's base level is consistent, and image is acquired with single light source. The raised terrain is constructed through raising soil to flat terrain so the actual sinkage is more than the sensor detection value. The hollow terrain is constructed through digging soil form flat terrain so the actual sinkage is less than the sensor detection value. The rough terrain is constructed through digging different volume of soil form flat terrain in different position so the actual sinkage is less than the sensor detection value.

The results of terrain adaptability testing experiments are shown in Fig. 8. Fig. 8 shows the comparison between the sinkage depth value detected by displacement sensor (DSDV) with the sinkage depth value detected by our method (MSDV). The results can correspond to experimental design. Thus, the sinkage detection method proposed in this paper is reliable, it can detect the sinkage of the wheel effectively when the rover is moving on all kinds of terrain conditions. 


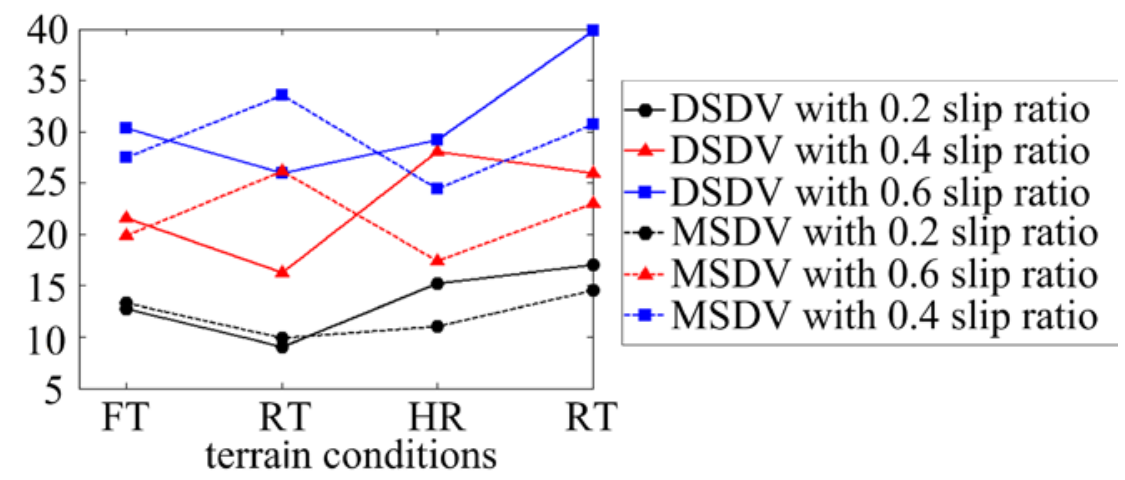

Fig. 8. Comparison of the sinkage depth

The Algorithm Precision Testing Experiments. The terrain's condition is flat terrain, the wheel speed is $10 \mathrm{~mm} / \mathrm{s}$, the vertical loads are $30 \mathrm{~N}$ and $90 \mathrm{~N}$, and the slip ratios are 0-0.7 in each load condition. There are 16 experiments.

TIAs are measured by manual way to know the accurate values. The relative errors of sinkage (RES) are shown in Table 2 and 3. It can be discovered from the table that the relative errors of the sinkage depth (REDS) are around 10\%, the relative errors of entrance angle (REEA) and the relative errors of departure angle (REDA) are around 5\%, without individual points which are in the small slip ratios.

Table.2 The relative errors of wheel sinkage with $30 \mathrm{~N}$ vertical load

\begin{tabular}{ccccccccc}
\hline slip ratio & 0 & 0.1 & 0.2 & 0.3 & 0.4 & 0.5 & 0.6 & 0.7 \\
\hline REDS [\%] & 2.54 & 0.27 & 2.29 & 2.13 & 1.35 & 4.03 & 2.89 & 8.70 \\
REEA [\%] & 19.74 & 11.81 & 1.14 & 3.47 & 4.06 & 0.18 & 2.44 & 1.84 \\
REDA [\%] & 4.83 & 2.33 & 1.09 & 0.42 & 0.44 & 1.61 & 1.60 & 1.15 \\
\hline
\end{tabular}

Table. 3 The relative errors of wheel sinkage with $90 \mathrm{~N}$ vertical load

\begin{tabular}{ccccccccc}
\hline slip ratio & 0 & 0.1 & 0.2 & 0.3 & 0.4 & 0.5 & 0.6 & 0.7 \\
\hline REDS [\%] & 2.70 & 0.48 & 1.76 & 3.33 & 5.75 & 4.10 & 9.13 & 2.48 \\
REEA [\%] & 0.73 & 3.61 & 2.53 & 0.67 & 0.36 & 3.65 & 2.93 & 2.35 \\
REDA [\%] & 3.14 & 4.11 & 2.75 & 1.20 & 0.47 & 1.70 & 3.77 & 3.82 \\
\hline
\end{tabular}

With analysis of the sinkage value under different slip ratios and vertical loads, it can be found that the relative errors are greater when the sinkage is smaller. The relative errors of wheel sinkage change along with sinkage depth are shown in Fig. 9. When the sinkage depth is above $5 \mathrm{~mm}$, the relative errors of the sinkage depth are around $10 \%$ and the relative errors of terrain interface angles are around $5 \%$. So the validity of this algorithm is intuitively clear.

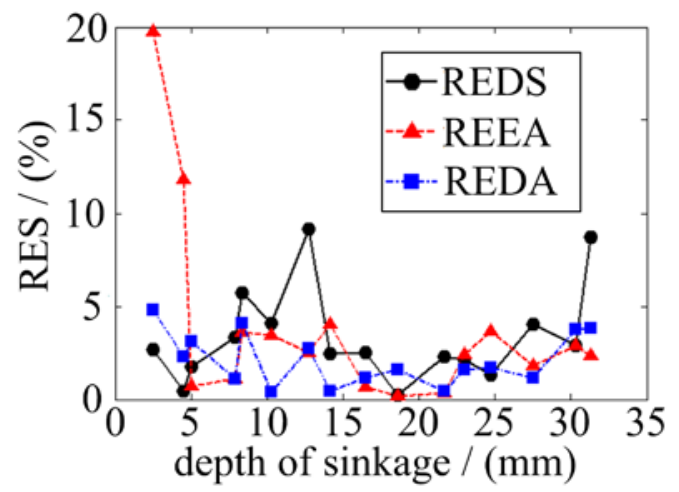

Fig. 9. The relative errors of wheel sinkage. 


\section{Conclusion}

This paper does some study on the wheel sinkage calculation model. The least square method is used to fit the wheel-soil boundary equivalent line, the mathematical calculation method for wheel sinkage is proposed according to the wheel-soil features. The wheel-soil interaction area image's color space RGB is transformed to HSI, and the image is processed to binary image according to the value scope of $\mathrm{H}$ and $\mathrm{S}$. Then the wheel-soil boundary is extracted, combining the coordinates' information of the points which are in the wheel-soil boundary with the math equations which are acquired from the wheel sinkage model. The results of the experiments indicate that the method is applicable to all terrain conditions. The relative errors of the sinkage depth are around $10 \%$, and the relative errors of terrain interface angles are around 5\%, when the actual sinkage depth is above $5 \mathrm{~mm}$.

The method proposed in this paper has lower accuracy, when the sinkage depth is less than 5mm, the future work is to research better extraction method to improve the accuracy of the sinkage detection. What's more, how to detect the wheel sinkage when the position of the camera relative to the wheel is changing or the image illumination is poor is the issue that needs to be solved.

\section{References}

[1] J. Y. Wong: Theory of ground vehicles. John Wiley \& Sons, New York (2001).

[2] K. Yoshida, H. Hamano: Motion dynamics of a rover with slip-based traction model. Robotics and Automation, 2002. Proceedings. ICRA'02. IEEE International Conference on. Vol. 3. IEEE (2002).

[3] L. Ding, H. B. Gao, Z. Q. Deng, et al: An approach of identifying mechanical parameters for lunar soil based on integrated wheel-soil interaction terramechanics model of rovers. Acta Aeronautica et Astronautica Sinica Vol .32 (2011), p. 1112-1123.

[4] K. Iagnemma, C. Brooks, S. Dubowsky: Visual, tactile, and vibration-based terrain analysis for planetary rovers.Aerospace Conference, 2004. Proceedings. 2004 IEEE. Vol. 2. IEEE(2004).

[5] G. Reina, L. Ojeda, A. Milella: Wheel slippage and sinkage detection for planetary rovers. IEEE IASME Transactions on Mechatronics Vol. 11(2006), p. 185-195.

[6] G. Reina, A. Milella, F. W. Panella: Vision-Based Wheel Sinkage Estimation for Rough-Terrain Mobile Robots. Mechatronics and Machine Vision in Practice, 2008. M2VIP 2008. 15th International Conference on. IEEE (2008).

[7] C. A. Brooks, K. D. Iagnemma, S. Dubowsky: Visual wheel sinkage measurement for planetary rover mobility characterization. Autonomous Robots Vol. 21 (2006), p. 55-64.

[8] B. Liu, L. Wang, P. Y. Cui: Visual wheel sinkage measurement for a lunar rover. Comuuter Measurement \& Control Vol. 16(2008), p. 1809-1818.

[9] L. Wang, B. X. Dai, H. H. Ju: Homography-based visual measurement of wheel Sinkage for a lunar rover. Journal of Astronautics Vol. 32(2011), p. 1701-1707.

[10]G. M. Hegde, C. Ye, C. A. Robinson, et al: Computer-vision-based wheel sinkage estimation for robot navigation on lunar terrain [J]. IEEE/ASME Transactions on Mechatronics Vol. 18 (2013), p. 1346-1356. 